\title{
Implementation of the Homotopy Perturbation Sumudu Transform Method for Solving Klein-Gordon Equation
}

\author{
Amr M. S. Mahdy', Adel S. Mohamed', Ahmad A. H. Mtawa' \\ ${ }^{1}$ Department of Mathematics, Faculty of Science, Zagazig University, Zagazig, Egypt \\ ${ }^{2}$ Department of Mathematics, Faculty of Science, Benghize University, Almarj, Libya \\ Email: amr-mahdy85@yahoo.com, 3adel@live.nl, hussanahmad65@yahoo.com
}

Received 16 February 2015; accepted 23 March 2015; published 24 March 2015

Copyright (C) 2015 by authors and Scientific Research Publishing Inc.

This work is licensed under the Creative Commons Attribution International License (CC BY). http://creativecommons.org/licenses/by/4.0/

\section{(c) (i) Open Access}

\section{Abstract}

This paper extends the homotopy perturbation Sumudu transform method (HPSTM) to solve linear and nonlinear fractional Klein-Gordon equations. To illustrate the reliability of the method, some examples are presented. The convergence of the HPSTM solutions to the exact solutions is shown. As a novel application of homotopy perturbation sumudu transform method, the presented work showed some essential difference with existing similar application four classical examples also highlighted the significance of this work.

\section{Keywords}

Mittag-Leffler Functions, Caputo Derivative, Sumudu Transform, Homotopy Perturbation Method, Klein-Gordon Equation

\section{Introduction}

Nonlinear phenomena that appear in many areas of scientific fields such as solid state physics, plasma physics, fluid dynamics, mathematical biology and chemical kinetics are modeled in terms of nonlinear partial differential equations and in many scientific and engineering applications one of the corner stones of modeling are partial differential equations. For example, the Klein-Gordon equation which is of the form

$$
w_{t t}(x, t)+b w(x, t)+g(w(x, t))=f(x, t)
$$

with initial conditions 


$$
w(x, 0)=h(x), \quad w_{t}(x, 0)=k(x)
$$

appears in modeling of problems in quantum field theory, relavistic physics, dispersive wave phenomena, plasma physic, nonlinear optics and applied physical sciences. The complexity of the equations though requires the use of numerical and analytical methods in most cases. A broad class of analytical solution and numerical solution methods were used to handle these problems. The topic of fractional partial differential equations has attracted a great atteation in the recent years. There are several analytical have been presented in the literature to solve fractional partial differential equations (FPDEs), such as the Fourier transform method [1], the fractional Greens function method [2], the Mellin transform method and the Laplace transform method [3] [4], the Sumudu transform method [5].

Recently, several numerical methods have been introduced for this purpose, such as: the homotopy perturbation method (HPM) has first proposed by He [6]-[8], the Modified homotopy perturbation method (MHPM) [9], the differential transform method (DTM) [10], the variational iteration method (VIM) [11] [12], the homotopy analysis method (HAM) [13] [14], the Sumudu decomposition method [15], the Adomian decomposition method [16] [17].

The homotopy perturbation method (HPM) is extended to drive the exact solutions for linear (nonlinear) ordinary (partial) differential equations of fractional order. The homotopy perturbation method is also combined with the vartional iteration method [18], to produce ahighly effective technique for handling many nonlinear problems. An also the homotopy perturbation method (HPM) is also combined with the laplace transform method [19]. The advantage of this methods for obtaining exact and approximate solutions for nonlinear equations.

The homotopy perturbation method (HPM) was also investigated by many researchers to handle partial differential equations arising in science and engineering [20] [21]. In addition, some numerical methods use a combination of utilizing specific transformation and obtaining series with converge to the exact solutions. An example of such a method is homotopy analysis Sumudu transform method (HASTD) which is a combination of the homotopy analysis method and the Sumudu transformation method [22]. Another such a combination is the which is the Sumudu decomposition method (SDM), which is constructed by combining two powerful methods, namely, the Sumudu transform method and Adomian decomposition method [23]. An efficent such approach is proposed combining the Sumudu transformation method with the homotopy perturbation method, which gives a new method called the homotopy perturbation Sumudu transform method (HPSTM) [24]. Recently, the homotopy perturbation Sumudu transform method (HPSTM) is frequently used for solving linear and nonlinear equations which are PDEs of integer order to obtain the exact solution.

In this paper, we applied homotopy perturbation Sumudu transform method (HPSTM) to obtain the analytical exact and approximate solutions for the fractional Klein-Gordon equation with time-fractional derivatives of the form:

$$
\begin{gathered}
\frac{\partial^{\alpha}}{\partial t^{\alpha}} w(x, t)+b w(x, t)+g(w(x, t))=f(x, t), \\
w(x, 0)=h(x), w_{t}(x, 0)=k(x),
\end{gathered}
$$

where $\alpha$ is parameters describing the order of the time fractional derivatives of $w(x, t)$, respectively, and they setisfy $1<\alpha \leq 2, b, g$ is constants, $h(x)$ and $k(x)$ is the initial conditions and $f(x, t)$ is is the source term and try to show the convergence of homotopy perturbation Sumudu transform method in solving this equation.

The paper is organized as follows: in Section 2, we recall some definitions of fractional calculus theory. In Section 3, we describe the homotopy perturbation Sumudu transform method. In Section 4, contains the main results and an examples to show the efficiency of using HPSTM to solve fractional-time Klein-Gordon equations. Conclusions are given in Section 5.

\section{Basic Definitions of Fractional Calculus}

In this section, we mention the following basic definitions and properties of the fractional calculus theory and Sumudu transform.

Definition 1 The Riemann-Liouville fractional integral operator of order $\alpha>0$, of a function $f(t) \in C_{\mu}$, 
$\mu \geq-1$, is defined as:

$$
\begin{aligned}
& J^{\alpha} f(t)=\frac{1}{\Gamma(\alpha)} \int_{0}^{t}(t-\tau)^{\alpha-1} f(\tau) \mathrm{d} \tau, \\
& J^{0} f(t)=f(t) .
\end{aligned}
$$

Definition 2 The fractional derivative of $f(t)$ in the Caputo sense is defined as [13] [18]

$$
\begin{aligned}
D_{t}^{\alpha} f(t) & =J^{m-\alpha} D^{n} f(t) \\
& =\frac{1}{\Gamma(n-\alpha)} \int_{0}^{t}(t-\tau)^{m-\alpha-1} f^{(m)}(\tau) \mathrm{d} \tau,
\end{aligned}
$$

for $m-1<\alpha \leq m, m \in N, t>0$ and $\Gamma(\alpha)$ is the Gamma function.

Definition 3 The Mittag-Leffler function which is ageneralization of exponential function (see [25]) is defined as:

$$
\begin{gathered}
E_{\alpha}(t)=\sum_{j=0}^{\infty} \frac{t^{j}}{\Gamma(\alpha j+1)}, \quad \alpha>0, \quad t \in \mathbb{C} \\
E_{\alpha, \beta}(t)=\sum_{j=0}^{\infty} \frac{t^{j}}{\Gamma(\alpha j+\beta)}, \quad \alpha, \beta>0, \quad t \in \mathbb{C}
\end{gathered}
$$

Some special cases of the Mittag-Leffler function are as follows:

1. $E_{1}(t)=\mathrm{e}^{t}$

2. $E_{\alpha, 1}(t)=E_{\alpha}(t)$

Definition 4 The Sumudu transform is defined over the set of functions:

$$
A=\left\{f(t)\left|\exists M, \tau_{1}, \tau_{2}>0,\right| f(t) \mid<M \mathrm{e}^{\frac{|t|}{\tau_{j}}} \text { if } t \in(-1)^{j} \times[0, \infty)\right\}
$$

by the following formula:

$$
\bar{f}(u)=\mathbf{S}[f(t)]=\int_{0}^{\infty} f(u t) \mathrm{e}^{-t} \mathrm{~d} t, \quad u \in\left(\tau_{1}, \tau_{2}\right)
$$

Some special properties of the Sumudu transform are as follows:
1. $S[1]=1$;
2. $S\left[\frac{t^{m}}{\Gamma(m+1)}\right]=u^{m} ; m>0$;

Other properties of the Sumudu transform can be found in [26].

Definition 5 The Sumudu transform of the Caputo fractional derivative is defined as follows [5]:

$$
\mathbf{S}\left[D_{t}^{\alpha} f(x, t)\right]=u^{-\alpha} \mathbf{S}[f(x, t)]-\sum_{k=0}^{m-1} u^{-\alpha+k} f^{(k)}(0+), \quad m-1<\alpha \leq m
$$

\section{The Homotopy Perturbation Sumudu Transform Method (HPSTM)}

To illustrate the basic idea of this method, we consider a general fractional partial differential equation with the initial condition of the form:

$$
D_{t}^{\alpha} w(x, t)+\mathbf{L} w(x, t)+\mathbf{N} w(x, t)=g(x, t)
$$

with $m-1<\alpha \leq m$, and subject to the initial condition

$$
\frac{\partial^{s} w(x, t)}{\partial t^{s}}=w^{s}(x, 0), \quad s=0,1,2, \cdots, m-1
$$


where $D_{t}^{\alpha} w(x, t)$ is the Caputo fractional derivative of the function $w(x, t), g(x, t)$ is the source term, $\mathbf{L}$ is the linear differential operator and $\mathbf{N}$ is the general nonlinear differential operator.

Applying the Sumudu transform (denoted in this paper by $\mathbf{S}$ ) on both sides of Equation (11), we get

$$
\mathbf{S}\left[D_{t}^{\alpha} w(x, t)\right]+\mathbf{S}[\mathbf{L} w(x, t)]+\mathbf{S}[\mathbf{N} w(x, t)]=\mathbf{S}[g(x, t)]
$$

Using the differentiation property of the Sumudu transform and the initial conditions in Equation (12), we have

$$
\mathbf{S}[w(x, t)]=\sum_{k=0}^{m-1} u^{-\alpha+k} w^{(k)}(x, 0)+u^{\alpha} \mathbf{S}[g(x, t)]-u^{\alpha} \mathbf{S}[\mathbf{L} w(x, t)+\mathbf{N} w(x, t)]
$$

Operating with the Sumudu inverse on both sides of Equation (14) gives

$$
w(x, t)=G(x, t)-\mathbf{S}^{-1}\left[u^{\alpha} \mathbf{S}[\mathbf{L} w(x, t)+\mathbf{N} w(x, t)-g(x, t)]\right]
$$

where $G(x, t)$ represent the prescribed initial conditions. Now we apply the HPM.

$$
w(x, t)=\sum_{n=0}^{\infty} p^{n} w_{n}(x, t)
$$

and the nonlinear term can be decomposed as

$$
\mathbf{N} w(x, t)=\sum_{n=0}^{\infty} p^{n} A_{n}
$$

for some Adomian's polynomials $A_{n}$ that are given by [27]

$$
A_{n}=\frac{1}{n !} \frac{\mathrm{d}^{n}}{\mathrm{~d} p^{n}}\left[\mathbf{N}\left(\sum_{i=0}^{\infty} p^{i} w_{i}\right)\right]_{p=0}, \quad n=0,1,2, \cdots
$$

Substituting Equation (16) and Equation (17) in Equation (15), we get

$$
\sum_{n=0}^{\infty} p^{n} w_{n}(x, t)=G(x, t)-p\left(\mathbf{S}^{-1}\left[u^{\alpha} \mathbf{S}\left[\mathbf{L}\left(\sum_{n=0}^{\infty} p^{n} w_{n}(x, t)\right)+\sum_{n=0}^{\infty} p^{n} A_{n}-g(x, t)\right]\right]\right)
$$

Equating the terms with identical powers of $p$, we can obtain a series of equations as the follows:

$$
\begin{aligned}
& p^{0}: w_{0}(x, t)=G(x, t), \\
& p^{1}: w_{1}(x, t)=-\mathbf{S}^{-1}\left[u^{\alpha} \mathbf{S}\left[\mathbf{L} w_{0}(x, t)+A_{0}-g(x, t)\right]\right], \\
& p^{2}: w_{2}(x, t)=-\mathbf{S}^{-1}\left[u^{\alpha} \mathbf{S}\left[\mathbf{L} w_{1}(x, t)+A_{1}-g(x, t)\right]\right], \\
& \text { and similarly } \\
& p^{n}: w_{n}(x, t)=-\mathbf{S}^{-1}\left[u^{\alpha} \mathbf{S}\left[\mathbf{L} w_{n-1}(x, t)+A_{n-1}-g(x, t)\right]\right],
\end{aligned}
$$

proceeding in the same manner, the rest of the components $w_{n}(x, t)$ can be completely found and the series solution is thus entirely determined. We approximate the analytical solution $w(x, t)$ by truncated series as:

$$
w(x, t)=\lim _{N \rightarrow \infty} \sum_{n=0}^{N} p^{n} w_{n}(x, t)
$$

\section{Applications}

In this section, in order to asses the applicability and the accuracy of the fractional homotopy Sumudu transform method the following four examples.

Example 1 Consider the time-fractional partial differential Klein-Gordon equation

$$
D_{t}^{\alpha} w(x, t)=\frac{\partial^{2} w(x, t)}{\partial x^{2}}-w(x, t), \quad 1<\alpha \leq 2
$$


subject to the initial conditions

$$
w(x, 0)=0, \quad w_{t}(x, 0)=x
$$

Taking the Sumudu transform on both sides of Equation (22), thus we get

$$
\mathbf{S}\left[D_{t}^{\alpha} w(x, t)\right]=\mathbf{S}\left[D_{x}^{2} w(x, t)-w(x, t)\right]
$$

and

$$
u^{-\alpha} \mathbf{S}[w(x, t)]-\left(u^{-\alpha} w(x, 0)+u^{1-\alpha} \frac{\partial w(x, 0)}{\partial t}\right)=\mathbf{S}\left[D_{x}^{2} w(x, t)-w(x, t)\right]
$$

Using the property of the Sumudu transform and the initial condition in Equation (23), we have

$$
\mathbf{S}[w(x, t)]=x t+u^{\alpha} \mathbf{S}\left[D_{x}^{2} w(x, t)-w(x, t)\right]
$$

Operating with the Sumudu inverse on both sides of Equation (24) we get

$$
[w(x, t)]=x t+\mathbf{S}^{-1}\left[u^{\alpha} \mathbf{S}\left[D_{x}^{2} w(x, t)-w(x, t)\right]\right]
$$

By applying the homotopy perturbation method, and substituting Equation (16) in Equation (25) we have

$$
\sum_{n=0}^{\infty} p^{n} w_{n}(x, t)=x t+p \mathbf{S}^{-1}\left[u^{\alpha} \mathbf{S}\left[\left(D_{x}^{2}-1\right)\left(\sum_{n=0}^{\infty} p^{n} w_{n}(x, t)\right)\right]\right]
$$

Equating the terms with identical powers of $p$, we get

$$
\begin{aligned}
& p^{0}: w_{0}(x, t)=x t, \\
& p^{1}: w_{1}(x, t)=\frac{-x t^{\alpha+1}}{\Gamma(\alpha+2)}, \\
& p^{2}: w_{2}(x, t)=\frac{x t^{2 \alpha+1}}{\Gamma(2 \alpha+2)}, \\
& p^{3}: w_{3}(x, t)=\frac{-x t^{3 \alpha+1}}{\Gamma(3 \alpha+2)}, \\
& \vdots \\
& p^{n}: w_{n}(x, t)=\frac{(-1)^{n} x t^{n \alpha+1}}{\Gamma(n \alpha+2)} .
\end{aligned}
$$

Thus the solution of Equation (22) is given by

$$
\begin{aligned}
w(x, t) & =\lim _{N \rightarrow \infty} \sum_{n=0}^{N} p^{n} w_{n}(x, t) \\
& =x\left(t-\frac{t^{\alpha+1}}{\Gamma(\alpha+2)}+\frac{t^{2 \alpha+1}}{\Gamma(2 \alpha+2)}-\frac{t^{3 \alpha+1}}{\Gamma(3 \alpha+2)}+\cdots\right) \\
& =x \sum_{n=0}^{\infty} \frac{(-1)^{n} x t^{n \alpha+1}}{\Gamma(n \alpha+2)} \\
& =x t E_{\alpha, 2}\left(-t^{\alpha}\right),
\end{aligned}
$$

If we put $\alpha \rightarrow 2$ in Equation (27) or solve Equations (22) and (23) with $\alpha=2$, we obtain the exact solution

$$
w(x, t)=x \sum_{n=0}^{\infty} \frac{(-1)^{n} x t^{n \alpha+1}}{\Gamma(n \alpha+2)}=x \sin t .
$$


Which is in full agreement with the result in Reference [28].

Example 2 Consider the inhomogeneous linear time-fractional partial differential Klein-Gordon equation

$$
D_{t}^{\alpha} w(x, t)=\frac{\partial^{2} w(x, t)}{\partial x^{2}}-w(x, t)+2 \sin x, \quad 1<\alpha \leq 2
$$

subject to the initial conditions

$$
w(x, 0)=\sin (x), \quad w_{t}(x, 0)=1
$$

Taking the Sumudu transform on both sides of Equation (28), thus we get

$$
\mathbf{S}\left[D_{t}^{\alpha} w(x, t)\right]=\mathbf{S}\left[D_{x}^{2} w(x, t)-w(x, t)+2 \sin (x)\right]
$$

and

$$
u^{-\alpha} \mathbf{S}[w(x, t)]-\left(u^{-\alpha} w(x, 0)+u^{1-\alpha} \frac{\partial w(x, 0)}{\partial t}\right)=\mathbf{S}\left[D_{x}^{2} w(x, t)-w(x, t)+2 \sin (x)\right]
$$

Using the property of the Sumudu transform and the initial condition in Equation (29), we have

$$
\mathbf{S}[w(x, t)]=\sin (x)+t+u^{\alpha} \mathbf{S}\left[D_{x}^{2} w(x, t)-w(x, t)+2 \sin (x)\right]
$$

Operating with the Sumudu inverse on both sides of Equation (30) we get

$$
[w(x, t)]=\sin (x)+t+\mathbf{S}^{-1}\left[u^{\alpha} \mathbf{S}\left[D_{x}^{2} w(x, t)-w(x, t)+2 \sin (x)\right]\right]
$$

By applying the homotopy perturbation method, and substituting Equation (16) in Equation (31) we have

$$
\sum_{n=0}^{\infty} p^{n} w_{n}(x, t)=\sin (x)+t+p \mathbf{S}^{-1}\left[u^{\alpha} \mathbf{S}\left[\left(D_{x}^{2}-1\right)\left(\sum_{n=0}^{\infty} p^{n} w_{n}(x, t)\right)+2 \sin (x)\right]\right]
$$

Equating the terms with identical powers of $p$, we get

$$
\begin{aligned}
& p^{0}: w_{0}(x, t)=\sin (x)+t, \\
& p^{1}: w_{1}(x, t)=\frac{-t^{\alpha+1}}{\Gamma(\alpha+2)}, \\
& p^{2}: w_{2}(x, t)=\frac{t^{2 \alpha+1}}{\Gamma(2 \alpha+2)}, \\
& p^{3}: w_{3}(x, t)=\frac{-t^{3 \alpha+1}}{\Gamma(3 \alpha+2)}, \\
& \vdots \\
& p^{n}: w_{n}(x, t)=\frac{(-1)^{n} t^{n \alpha+1}}{\Gamma(n \alpha+2)} .
\end{aligned}
$$

Thus the solution of Equation (36) is given by

$$
\begin{aligned}
w(x, t) & =\lim _{N \rightarrow \infty} \sum_{n=0}^{N} p^{n} w_{n}(x, t) \\
& =\sin (x)+t-\frac{t^{\alpha+1}}{\Gamma(\alpha+2)}+\frac{t^{2 \alpha+1}}{\Gamma(2 \alpha+2)}-\frac{t^{3 \alpha+1}}{\Gamma(3 \alpha+2)}+\cdots \\
& =\sin (x)+\sum_{n=0}^{\infty} \frac{(-1)^{n} x t^{n \alpha+1}}{\Gamma(n \alpha+2)} \\
& =\sin (x)+t E_{\alpha, 2}\left(-t^{\alpha}\right),
\end{aligned}
$$


If we put $\alpha \rightarrow 2$ in Equation (33) or solve Equations (28) and (29) with $\alpha=2$, we obtain the exact solution

$$
\begin{aligned}
w(x, t) & =\sin (x)+\sum_{n=0}^{\infty} \frac{(-1)^{n} x t^{n \alpha+1}}{\Gamma(n \alpha+2)} \\
& =\sin (x)+\sin t .
\end{aligned}
$$

Which is in full agreement with the result in Reference [28].

Example 3 Consider the non-linear time-fractional partial differential Klein-Gordon equation

$$
D_{t}^{\alpha} w(x, t)=\frac{\partial^{2} w(x, t)}{\partial x^{2}}-w^{2}(x, t)+2 x^{2}-2 t^{2}+x^{4} t^{4}, \quad 1<\alpha \leq 2
$$

subject to the initial conditions

$$
w(x, 0)=0, \quad w_{t}(x, 0)=0
$$

Taking the Sumudu transform on both sides of Equation (34), thus we get

$$
\mathbf{S}\left[D_{t}^{\alpha} w(x, t)\right]=\mathbf{S}\left[D_{x}^{2} w(x, t)-w^{2}(x, t)+2 x^{2}-2 t^{2}+x^{4} t^{4}\right]
$$

and

$$
u^{-\alpha} \mathbf{S}[w(x, t)]-\left(u^{-\alpha} w(x, 0)+u^{1-\alpha} \frac{\partial w(x, 0)}{\partial t}\right)=\mathbf{S}\left[D_{x}^{2} w(x, t)-w^{2}(x, t)+2 x^{2}-2 t^{2}+x^{4} t^{4}\right]
$$

Using the property of the Sumudu transform and the initial condition in Equation (35), we have

$$
\mathbf{S}[w(x, t)]=u^{\alpha} \mathbf{S}\left[D_{x}^{2} w(x, t)-w^{2}(x, t)+2 x^{2}-2 t^{2}+x^{4} t^{4}\right]
$$

Operating with the Sumudu inverse on both sides of Equation (36) we get

$$
[w(x, t)]=\mathbf{S}^{-1}\left[u^{\alpha} \mathbf{S}\left[D_{x}^{2} w(x, t)-w^{2}(x, t)+2 x^{2}-2 t^{2}+x^{4} t^{4}\right]\right]
$$

By applying the homotopy perturbation method, and substituting Equations (16) in (37) we have

$$
\sum_{n=0}^{\infty} p^{n} w_{n}(x, t)=p \mathbf{S}^{-1}\left[u^{\alpha} \mathbf{S}\left[D_{x}^{2}\left(\sum_{n=0}^{\infty} p^{n} w_{n}(x, t)\right)-\left(\sum_{n=0}^{\infty} p^{n} w_{n}(x, t)\right)^{2}+2 x^{2}-2 t^{2}+x^{4} t^{4}\right]\right]
$$

Equating the terms with identical powers of $p$, we get

$$
\begin{aligned}
& p^{0}: w_{0}(x, t)=0, \\
& p^{1}: w_{1}(x, t)=\frac{2 x^{2} t^{\alpha}}{\Gamma(\alpha+1)}, \\
& p^{2}: w_{2}(x, t)=\left[\frac{4 t^{2 \alpha}}{\Gamma^{2}(\alpha+1)}-\frac{4 x^{4} t^{3 \alpha}}{\Gamma^{3}(\alpha+1)}-\frac{2 t^{\alpha+2}}{\Gamma(\alpha+1)}+\frac{x^{4} t^{\alpha+4}}{\Gamma(\alpha+1)}\right],
\end{aligned}
$$

Thus the solution of Equation (34) is given by

$$
\begin{aligned}
w(x, t) & =\lim _{N \rightarrow \infty} \sum_{n=0}^{N} p^{n} w_{n}(x, t) \\
& =\frac{2 x^{2} t^{\alpha}}{\Gamma(\alpha+1)}+\frac{4 t^{2 \alpha}}{\Gamma^{2}(\alpha+1)}-\frac{4 x^{4} t^{3 \alpha}}{\Gamma^{3}(\alpha+1)}-\frac{2 t^{\alpha+2}}{\Gamma(\alpha+1)}+\frac{x^{4} t^{\alpha+4}}{\Gamma(\alpha+1)}+\cdots
\end{aligned}
$$

If we put $\alpha \rightarrow 2$ in Equation (39) or solve Equations (34) and (35) with $\alpha=2$, and so on, we can find that

$$
w_{n}(x, t)=0, \quad n>1
$$


we obtain the exact solution

$$
w(x, t)=x^{2} t^{2}
$$

Which is in full agreement with the result in Reference [28].

Example 4 Consider the one-dimensional linear inhomogeneous fractional Klein-Gordon equation

$$
D_{t}^{\alpha} w(x, t)=\frac{\partial^{2} w(x, t)}{\partial x^{2}}-w(x, t)+6 x^{3} t+6\left(x^{3}-6 x\right) t^{3}, \quad t>0, x \in \mathbb{R}, 1<\alpha \leq 2
$$

subject to the initial conditions

$$
w(x, 0)=0, \quad w_{t}(x, 0)=0
$$

Taking the Sumudu transform on both sides of Equation (40), thus we get

$$
\mathbf{S}\left[D_{t}^{\alpha} w(x, t)\right]=\mathbf{S}\left[D_{x}^{2} w(x, t)-w(x, t)+6 x^{3} t+6\left(x^{3}-6 x\right) t^{3}\right]
$$

and

$$
u^{-\alpha} \mathbf{S}[w(x, t)]-\left(u^{-\alpha} w(x, 0)+u^{1-\alpha} \frac{\partial w(x, 0)}{\partial t}\right)=\mathbf{S}\left[D_{x}^{2} w(x, t)-w(x, t)+6 x^{3} t+6\left(x^{3}-6 x\right) t^{3}\right]
$$

Using the property of the Sumudu transform and the initial condition in Equation (41), we have

$$
\mathbf{S}[w(x, t)]=u^{\alpha} \mathbf{S}\left[D_{x}^{2} w(x, t)-w(x, t)+6 x^{3} t+6\left(x^{3}-6 x\right) t^{3}\right]
$$

Operating with the Sumudu inverse on both sides of Equation (42) we get

$$
[w(x, t)]=\mathbf{S}^{-1}\left[u^{\alpha} \mathbf{S}\left[D_{x}^{2} w(x, t)-w(x, t)+6 x^{3} t+6\left(x^{3}-6 x\right) t^{3}\right]\right]
$$

By applying the homotopy perturbation method, and substituting Equation (16) in Equation (43) we have

$$
\sum_{n=0}^{\infty} p^{n} w_{n}(x, t)=p \mathbf{S}^{-1}\left[u^{\alpha} \mathbf{S}\left[\left(D_{x}^{2}-1\right)\left(\sum_{n=0}^{\infty} p^{n} w_{n}(x, t)\right)+6 x^{3} t+6\left(x^{3}-6 x\right) t^{3}\right]\right]
$$

Equating the terms with identical powers of $p$, we get

$$
\begin{aligned}
& p^{0}: w_{0}(x, t)=0 \\
& p^{1}: w_{1}(x, t)=\frac{6 x^{3} t^{\alpha+1}}{\Gamma(\alpha+2)}+\frac{6\left(x^{3}-6 x\right) t^{\alpha+3}}{\Gamma(\alpha+4)}, \\
& p^{2}: w_{2}(x, t)=-\left[\frac{6\left(x^{3}-6 x\right) t^{2 \alpha+1}}{\Gamma(2 \alpha+2)}+\frac{6\left(x^{3}-12 x\right) t^{2 \alpha+3}}{\Gamma(2 \alpha+4)}\right],
\end{aligned}
$$

Thus the solution of Equation (40) is given by

$$
\begin{aligned}
w(x, t) & =\lim _{N \rightarrow \infty} \sum_{n=0}^{N} p^{n} w_{n}(x, t) \\
& =\frac{6 x^{3} t^{\alpha+1}}{\Gamma(\alpha+2)}+\frac{6\left(x^{3}-6 x\right) t^{\alpha+3}}{\Gamma(\alpha+4)}-\left[\frac{6\left(x^{3}-6 x\right) t^{2 \alpha+1}}{\Gamma(2 \alpha+2)}+\frac{6\left(x^{3}-12 x\right) t^{2 \alpha+3}}{\Gamma(2 \alpha+4)}\right]+\cdots
\end{aligned}
$$

If we put $\alpha \rightarrow 2$ in Equation (45) or solve Equations (40) and (41) with $\alpha=2$, we obtain the exact solution

$$
w(x, t)=x^{3} t^{3}-0.0019047619 x^{3} t^{7}+0.01428571429 x t^{7}+\cdots
$$

Which is in full agreement with the result in Reference [29]. 
As it is presented above in Example 4 we obtained homotopy perturbation Sumudu transform solution of Equation (40) for values of $\alpha=2, \alpha=1.5, \alpha=1.75$. Figures 1-4 show the approximate solutions for Equation (40) obtained for the three different values of $\alpha$ using the homotopy perturbation Sumudu transform method (HPSTM). The values of $\alpha=2$ is the only case for which we know the exact solution $w(x, t)=x^{3} t^{3}$ and the results of (HPSTM) are in excellent agreement with the exact solution.

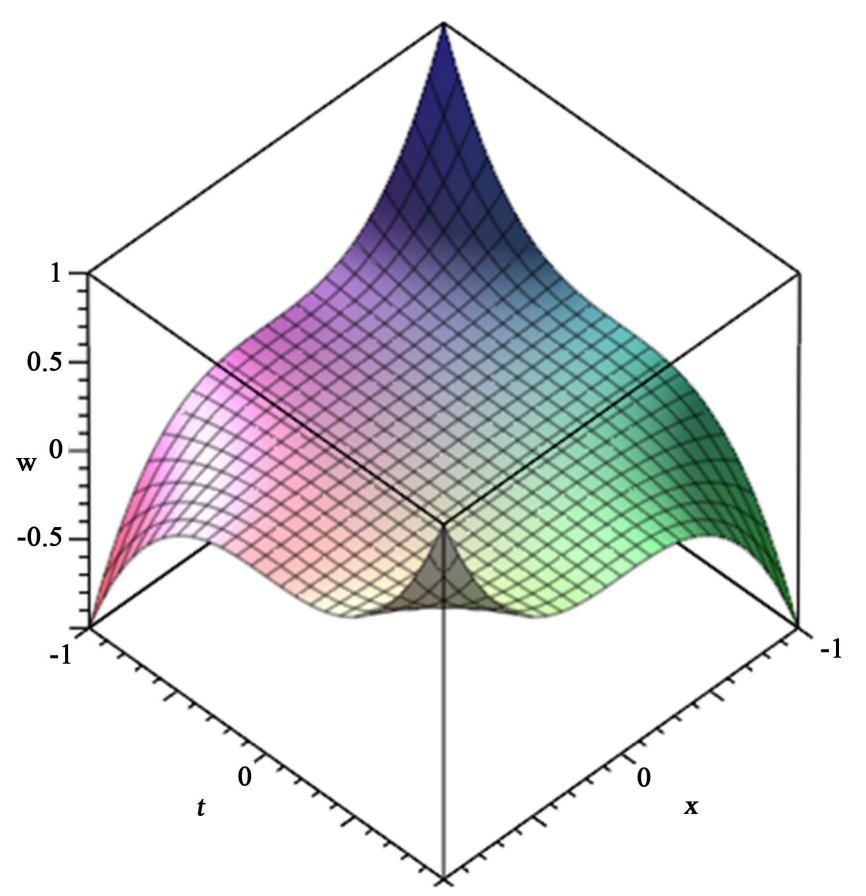

Figure 1. Profiles of $\mathrm{w}(\mathrm{x}, \mathrm{t})$ when $\alpha=2$ : Exact solution of (40).

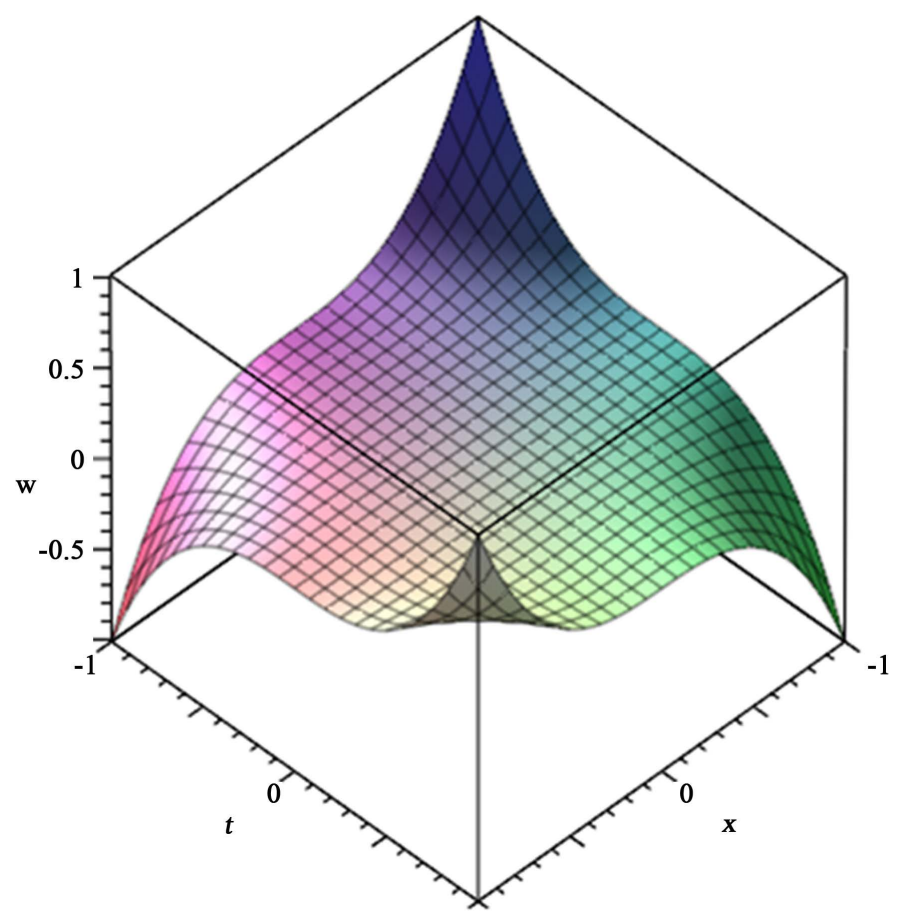

Figure 2. Profiles of w(x, t) when $\alpha=2$ : Approximate solution of (40). 


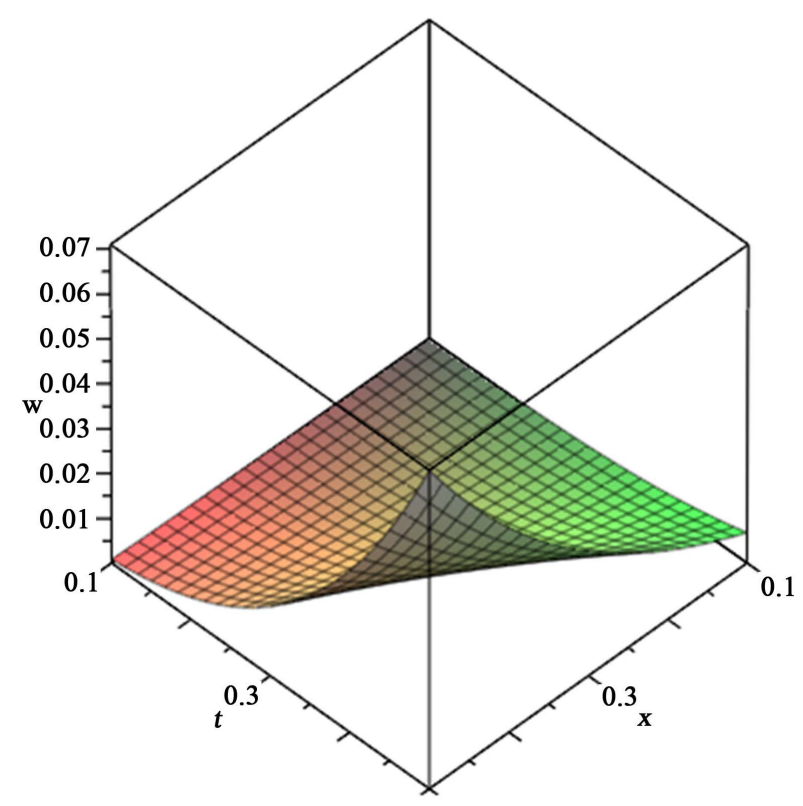

Figure 3. Profiles of $\mathrm{w}(\mathrm{x}, \mathrm{t})$ when $\alpha=1.5$ : Approximate solution of (40).

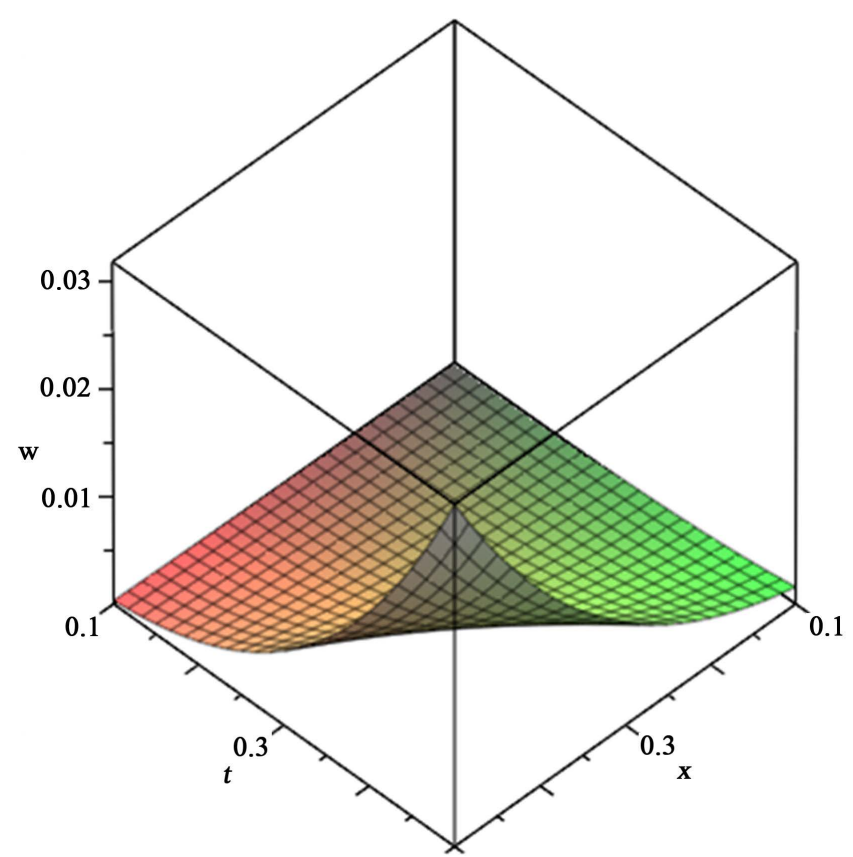

Figure 4. Profiles of $\mathrm{w}(\mathrm{x}, \mathrm{t})$ when $\alpha=1.75$ : Approximate solution of (40).

\section{Conclusion}

In this paper, we have introduced a combination of the homotopy perturbation method and the Sumudu transform method for time fractional problems. This combination builds a strong method called the HPSTD. This method has been successfully applied to one-dimensional fractional equations and also for problems of linear and nonlinear partial differential equations. The HPSTD is an analytical method and runs by using the initial conditions only. Thus, it can be used to solve equations with fractional and integer order with respect to time. An important advantage of the new approach is its low computational load. 


\section{References}

[1] Magin, R.L. and Ovadia, M. (2008) Modeling the Cardiac Tissue Electrode In-Terface Using Fractional Calculus. Journal of Vibration and Control, 14, 1431-1442. http://dx.doi.org/10.1177/1077546307087439

[2] Mainardi, F. (1995) Fractional Diffusive Waves in Viscoelastic Solids. In: Wegner, J.L. and Norwood, F.R., Eds., Nonlinear Waves in Solids, ASME Book No. AMR 137, Fairfield, 93-97.

[3] Odibat, Z. and Momani, S. (2007) A Reliable Treatment of Homotopy Perturbation Method for Klein-Gordon Equations. Physics Letters A, 365, 351-357. http://dx.doi.org/10.1016/j.physleta.2007.01.064

[4] Podlubny, I. (1999) Fractional Differential Equations: An Introduction to Fractional Derivatives, Fractional Equations, to Methods of Their Solution and Same of Their Applications. Academic Press, New York.

[5] Gupta, V.G. and Sharma, B. (2010) Application of Sumudu Transform in Reaction-Diffusion Systems and Nonlinear Waves. Applied Mathematical Sciences, 4, 435-446.

[6] He, J.H. (1998) Approximate Analytical Solution for Seepage Flow with Fractional Derivatives in Porous Media. Computer Methods in Applied Mechanics and Engineering, 167, 57-68. http://dx.doi.org/10.1016/S0045-7825(98)00108-X

[7] He, J.H. (2005) Limit Cycle and Bifurcation of Nonlinear Problems. Chaos, Solutions and Fractals, 24, 827-833. http://dx.doi.org/10.1016/j.chaos.2005.03.007

[8] He, J.H. (1997) A New Approach to Nonlinear Partial Differential Equations. Communications in Nonlinear Science and Numerical Simulation, 2, 230-235. http://dx.doi.org/10.1016/S1007-5704(97)90007-1

[9] Hesameddini, E. and Latifzadeh, H. (2011) An Optimal Choice of Initial Solutions in the Homotopy Perturbation Method. International Journal of Nonlinear Sciences and Numerical Simulation, 10, 1389-1398.

[10] Miller, K.S. and Ross, B. (1993) An Introduction to the Fractional Calculus and Fractional Differential Equations. John Wiley and Sons, New York.

[11] Bhalekar, S. and Daftardar-Gejji, V. (2008) New Iterative Method: Application to Partial Differential Equations. Applied Mathematics and Computation, 203, 778-783. http://dx.doi.org/10.1016/j.amc.2008.05.071

[12] Daftardar-Gejji, V. and Bhalekar, S. (2010) Solving Fractional Boundary Value Problems with Dirichlet Boundary Conditions Using a New Iterative Method. Computers \& Mathematics with Applications, 59, 1801-1809. http://dx.doi.org/10.1016/j.camwa.2009.08.018

[13] Arafa, A.A.M., Rida, S.Z. and Mohamed, H. (2011) Homotopy Analysis Method for Solving Biological Population Model. Communications in Theoretical Physics, 56, 797-800.

[14] Hilfe, R., Ed. (2000) Applications of Fractional Calculus in Physics. World Scientific, Singapore City.

[15] Eltayeb, H. and Kilicman, A. (2012) Application of Sumudu Decomposition Method to Solve Nonlinear System of Partial Differential Equations. Hindawi Publishing Corporation, Abstract and Applied Analysis, 2012, Article ID: 412948. http://dx.doi.org/10.1155/2012/412948

[16] Adomian, G. (1994) Solving Frontier Problems of Physics: The Decomposition Method. Kluwer Academic Publishers, Boston and London. http://dx.doi.org/10.1007/978-94-015-8289-6

[17] Cheng, J.F. and Chu, Y.M. (2011) Solution to the Linear Fractional Differential Equation Using Adomian Decomposition Method. Mathematical Problems in Engineering, 2011, 1-14. http://dx.doi.org/10.1155/2011/587068

[18] Noor, M.A. and Mohyud-Din, S.T. (2008) Variational Homotopy Perturbation Method for Solving Higher Dimensional Initial Boundary Value Problems. Mathematical Problems in Engineering, 2008, Article ID: 696734. http://dx.doi.org/10.1155/2008/696734

[19] Kumar, S., Yildirin, A. and Wei, L. (2012) A Fractional Model of the Diffusion Equation and Its Analytical Solution Using Laplace Transform. Scientia Iranica, 19, 1117-1123. http://dx.doi.org/10.1016/j.scient.2012.06.016

[20] Moustafa, O.L. (2003) On the Cauchy Problem for Some Fractional Order Partial Differential Equations. Chaos, Solitons \& Fractals, 18, 135-140. http://dx.doi.org/10.1016/S0960-0779(02)00586-6

[21] Rafei, M. and Ganji, D.D. (2006) Explicit Solutions of Helmholtz Equation and Fifth-Order KdV Equation Using Homotopy Perturbation Method. International Journal of Nonlinear Sciences and Numerical Simulation, 7, 321-329.

[22] Rathore, S., Kumar, D., Singh, J. and Gupta, S. (2012) Homotopy Analysis Sumudu Transform Method for Nonlinear Equations. International Journal of Industrial Mathematics, 4, 301-314.

[23] Kumar, D., Singh, J. and Rathore, S. (2012) Sumudu Decomposition Method for Nonlinear Equations. International Mathematical Forum, 7, 515-521.

[24] Karbalaie, A., Montazeri, M.M. and Muhammed, H.H. (2014) Exact Solution of Time-Fractional Partial Dioerential Equations Using Sumudu Transform. WSEAS Transactions on Mathematics, 13, 142-151.

[25] Kilbas, A.A., Saigo, M. and Saxena, R.K. (2004) Generalized Mittag-Leffler Function and Generalized Fractional 
Calculus Operators. Integral Transforms and Special Functions, 15, 31-49. http://dx.doi.org/10.1080/10652460310001600717

[26] Belgacem, F.B.M. and Karaballi, A.A. (2006) Sumudu Transform Fundamental Properties Investigations and Application. Journal of Applied Mathematics and Stochastic Analysis, 2006, 1-23. http://dx.doi.org/10.1155/JAMSA/2006/91083

[27] Ghorbani, A. (2009) Beyond Adomian Polynomials: He Polynomials. Chaos, Solitons \& Fractals, 39, $1486-1492$. http://dx.doi.org/10.1016/j.chaos.2007.06.034

[28] Marasi, H.R. and Karimi, S. (2014) Convergence of the Variational Iteration Method for Solving Fractional KleinGordon Equation. Journal of Mathematical and Computational Science, 4, 257-266.

[29] Turut, V. and Güzel, N. (2013) On Solving Partial Differential Equations of Fractional Order by Using the Variational Iteration Method and Multivariate Padé Approximations. European Journal of Pure and Applied Mathematics, 6, 147-171. 